\title{
Large dynamic range Atomic Force Microscope for overlay improvements
}

\author{
Stefan Kuiper*a, Erik Fritz, Will Crowcombe ${ }^{\mathrm{a}}$, Thomas Liebig ${ }^{\mathrm{a}}$, Geerten Kramer ${ }^{\mathrm{a}}$, Gert Witvoet ${ }^{\mathrm{a}}$, \\ Tom Duivenvoorde ${ }^{\mathrm{a}}$, Ton Overtoom ${ }^{\mathrm{a}}$, Ramon Rijnbeek ${ }^{\mathrm{a}}$, Erwin van Zwet ${ }^{\mathrm{a}}$, Anton van Dijsseldonk ${ }^{\mathrm{b}}$, \\ Arie den Boef ${ }^{\mathrm{b}}$, Marcel Beems ${ }^{\mathrm{b}}$, Leon Levasier ${ }^{\mathrm{b}}$

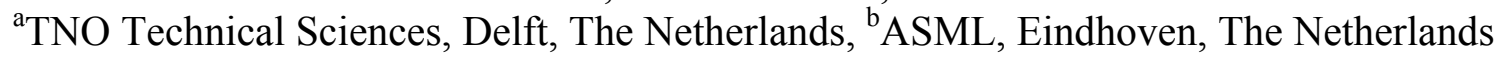

\begin{abstract}
Nowadays most overlay metrology tools assess the overlay performance based on marker features which are deposited next to the functional device features within each layer of the semiconductor device. However, correct overlay of the relatively coarse marker features does not directly guarantee correct overlay of the much smaller device features. This paper presents the development of a tool that allows to measure the relative distance between the marker and device features within each layer of the semiconductor device, which can be used to improve the overlay at device feature level. In order to be effective, the marker to device feature distance should be measured with sub-nanometer measurement uncertainty over several millimeters range. Furthermore, the tool should be capable of profiling the marker features to allows prediction of the location interpretation of the optical diffraction based alignment sensors, which are sensitive for potential asymmetry of the marker features.

To enable this, a highly stable Atomic Force Microscope system is being developed. The probe is positioned relative to the wafer with a $6 \mathrm{DOF}$ controlled hexapod stage, which has a relatively large positioning range of $8 \times 8 \mathrm{~mm}$. The position and orientation of this stage is measured relative to the wafer using 6 interferometers via a highly stable metrology frame. A tilted probe concept is utilized to allow profiling of the high aspect ratio marker and device features. Current activities are aimed at demonstrating the measurement capabilities of the developed AFM system.
\end{abstract}

Keywords: Scanning probe microscopy, SPM, Atomic Force Microscopy, AFM, overlay

\section{INTRODUCTION}

Semiconductor devices are manufactured in sequential steps, where in each step a pattern of material is deposited on the wafer. In order for the eventual semiconductor device to function correctly, the different patterns must be aligned correctly. The degree to which these patterns are aligned is referred to as overlay. Incorrect overlay can impact the functionality of the eventual semiconductor device.

Most overlay metrology tools evaluate the overlay performance based on marker features which are printed along with the device features within each layer, as depicted in Figure 1. These marker features typically consist of line features with relatively larges pitches in the order of several micrometers, which makes them detectable with optical methods. However, due to various errors, correct overlay of marker features does not directly guarantee correct overlay of the much finer functional device features, which nowadays can have line pitches down to a few dozens of nanometers. This is mainly due to lens aberrations within the lithography machine which affect the smaller line pitch features in a different way than the coarser marker features. The resulting position error between the device features and the markers is a potential source of overlay error. Within the near future the overlay budgets will go down to only a few nanometers, which makes it more and more relevant to compensate for this overlay error contribution.

*stefankuiper@tno.nl, phone number: +31 888664806

Metrology, Inspection, and Process Control for Microlithography XXX, edited by Martha I. Sanchez, Vladimir A. Ukrainstev Proc. of SPIE Vol. 9778, 97781B · C 2016 SPIE · CCC code: 0277-786X/16/\$18 · doi: 10.1117/12.2218249 
In [1] CD-SEM is proposed to measure in-die overlay via dedicated targets or directly on device features. This paper presents a novel method for improving overlay at device level based on Atomic Force Microscopy (AFM). In this concept AFM is used to accurately map the location of device features with respect to the alignment markers within each layer of the semiconductor device, as indicated in Figure 1. These AFM measurements will be done after etching of the wafer. Based on these measurements the relative position errors can be determined within each layer, which can be used the to improve the overlay within the lithography process. The major advantage of AFM is the fact that it not only allows to accurately measure the relative distance between the different features within each layer, but also directly provides profile information of these features. This profile information allows to determine the locations of both the top and the bottom of the features, and provides a way to compensate for overlay errors induced by asymmetry of the marker features.

This AFM concept is intended as an offline metrology tool, mapping the location of the device locations on several sample wafers during the initialization phase of the lithography process. Since the position errors of the device features with respect to the marker features are mainly stemming from the stationary lens aberrations in the lithography machine, these position errors can be considered largely systematic. Therefore, it is sufficient to measure these position errors on a few sample wafers, after which the offset can be corrected for in future batches.

In this paper the operational concept of using AFM as a means for improvinge overlay is explained and the system concept of the developed highly stable AFM system is presented.

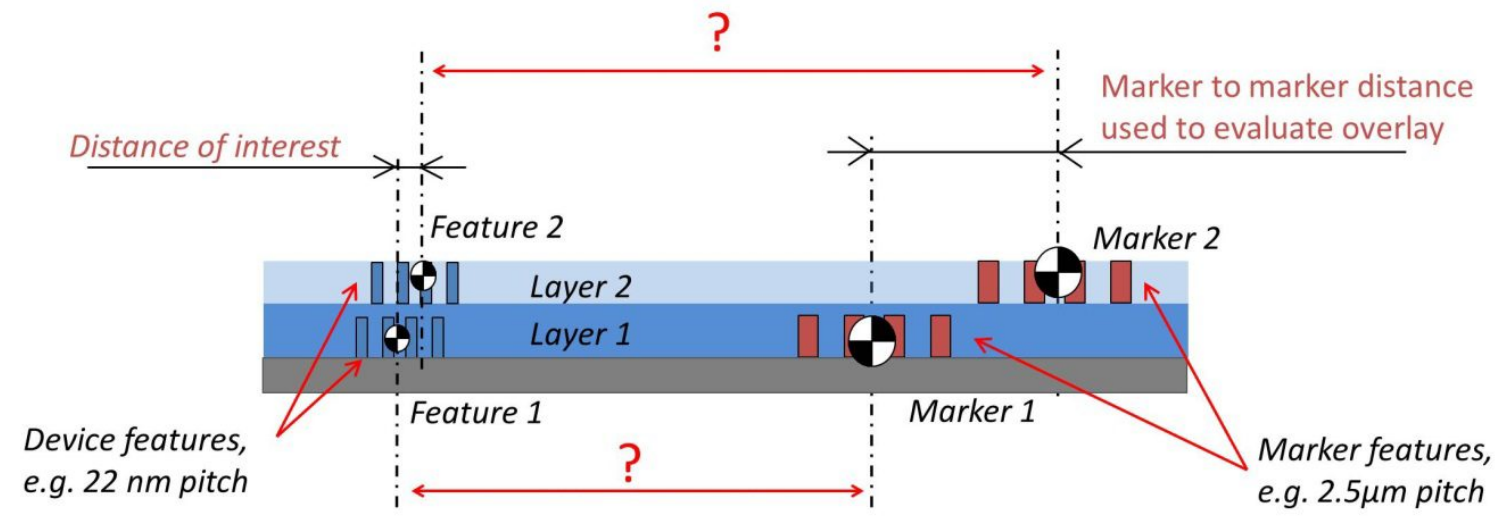

Figure 1. Conceptual drawing of two layers within a semiconductor device, with device features and marker features in each layer. The intra-layer overlay is assessed via marker features, while the relative position of the device features is actually determining the functionality of the semiconductor device. The idea of this metrology concept is to measure the unknown inter-layer marker to device feature distance marked by the red (?), which information can be used to improve the overlay at device feature level.

\section{OPERATIONAL CONCEPT}

\subsection{Overall measurement procedure}

Figure 2 depicts the measurement situation on the wafer level after etch, with the device feature areas marked in gray and the marker features in the scribe lane. In this example the distance of interest is the position of a set of line features in the middle of the device feature area with respect to the marker grid. For these sets of line features the distance in the axis orthogonal to the feature lines is the most critical for the overlay performance. Depending on the overall die-size this distance can go up to several millimeters. For effective overlay improvement this distance needs to be measured with sub-nanometer measurement uncertainty.

Although the marker to device feature distance could be measured in absolute units, it makes most sense to measure the distance relative to the marker grid. This is because the marker grid serves as a reference for the subsequent lithography steps to account for possible scaling and position errors. Furthermore, as also shown in Figure 2, the device features of interest may not be directly in line with the marker features. This causes an Abbe-offset which leads to a position error when the orientation of the wafer is not fully known with respect to the axis of the metrology tool. To compensate for the 
abovementioned error sources the device feature position is to be measured with respect to at least three marker features, of which at least one of the marker features is not directly in line with the other marker features, as shown in Figure 2. Subsequently, the marker to feature distance is scaled based on the measured marker to marker distances:

$$
\overline{X_{1}}=\left(X_{1 m}-\delta X_{3 m} \cdot \frac{Y_{1}}{Y_{3}}\right) \cdot \frac{X_{2 r}}{X_{2 m}}
$$

with all parameters as defined in Figure 2, and $X_{2 r}$ the distance between marker 1 and marker 2 according to a reference map. Hence, the location of the device feature is measured with respect in the coordinates of the alignment marker grid. Since these markers locations are also measured within the lithography machine this allows for compensation of scale mismatches due to for instance calibration errors, orthogonality errors of the metrology coordinate system and for temperature variations of the wafer.

Although the marker to device feature distance needs to be measured with sub-nanometer measurement uncertainty, the position variation of each point on the line is dominated by the Line Edge Roughness (LER), which can exceed several nanometers. The compensate for the line-edge roughness, several line traces are taken of each marker and device feature such that the random effects of the line-edge roughness average out. Hence, the marker and feature locations are evaluated over an certain area with sufficient line crossings orthogonal to the distance of interest.

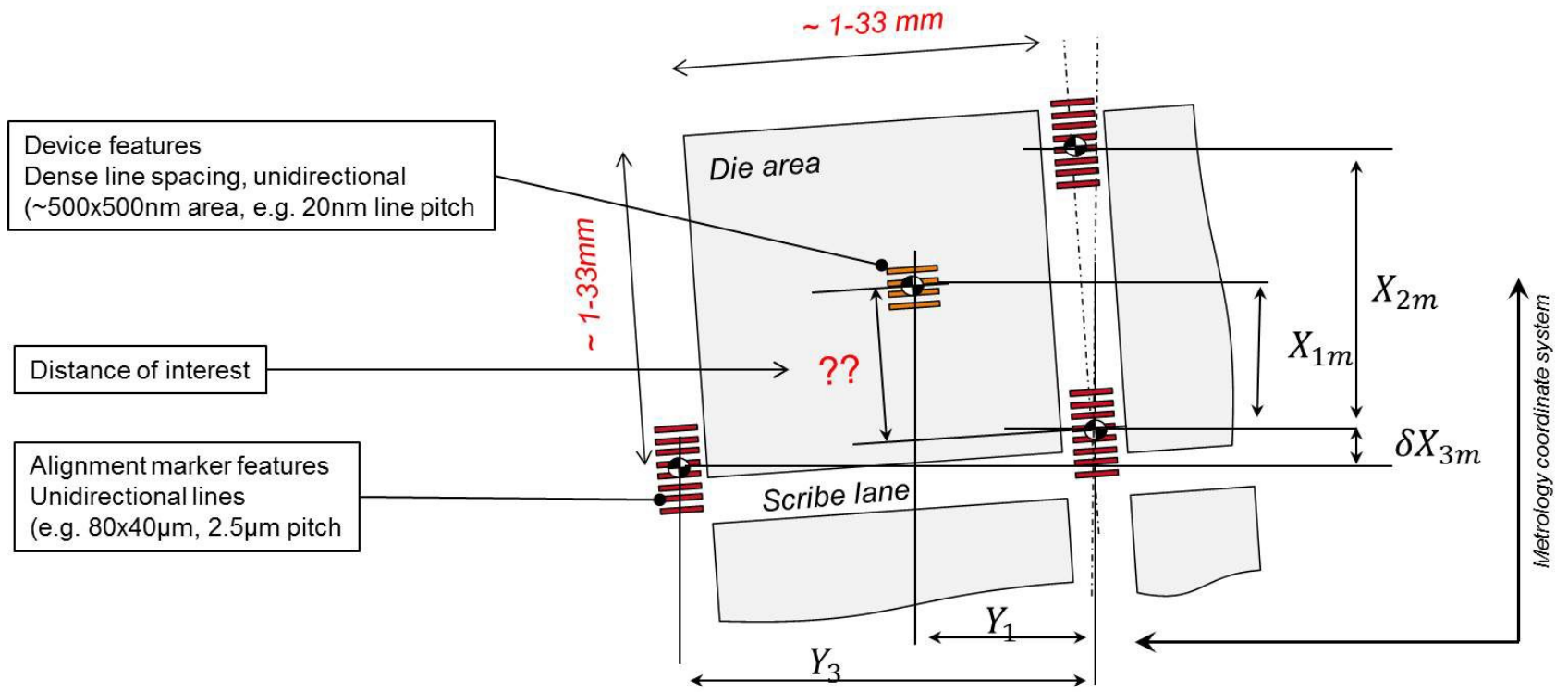

Figure 2: Situation sketch of the interlayer measurement of the device feature position with respect to the alignment marker grid.

\subsection{Why AFM?}

Within this concept Atomic Force Mircoscopy (AFM) techniques are used to measure the location of both the marker and device features within each layer. AFM is already used for several applications within the semiconductor industry, such as CD, and sidewall angle measurements [2,3], and defect inspection [4]. The major advantages of AFM over other imaging methods are the direct nature of the measurement, which allows to determine the position of each feature without extensive calibration of the metrology tool. AFM system are capable of reaching very high imaging resolution at (sub-)nanometer level which is needed to achieve the required measurement accuracy. With the right AFM imaging modes, AFM is non-destructive to the sample which allows this measurement to be performed on production wafers, and guarantees high integrity of the measurement. Furthermore, AFM allows to fully trace the profile of each alignment marker, which can be used to match the marker location interpretation of the AFM based metrology tool with other metrology and alignment tools to compensate for instance for asymmetry of the marker features. 


\subsection{Overcoming the AFM probe convolution problem}

One major challenge with applying AFM on semiconductor features is caused by the relatively steep sidewall angles of these features, which are typically around 90 degrees. The cone-angle of a AFM probes goes down to about 10 degrees for high aspect ratio probes. Hence, when such probe is positioned orthogonal to the sample surface it is incapable of reaching the sidewalls of the feature, and the resulting feature image is a convolution of the probe shape with only the top part of the feature. As a result only the location of the upperpart of the feature can be measured, while in some causes rather the location of the bottom or middle part of the feature are of interest.

Additionally, the convolution of the AFM probe results in a broadening of the features as they appear in the measurement. In determining the location of the center of gravity of a feature this causes a problem when the broadening is not equal on both sides, which may be caused by asymmetry of the probe or feature.

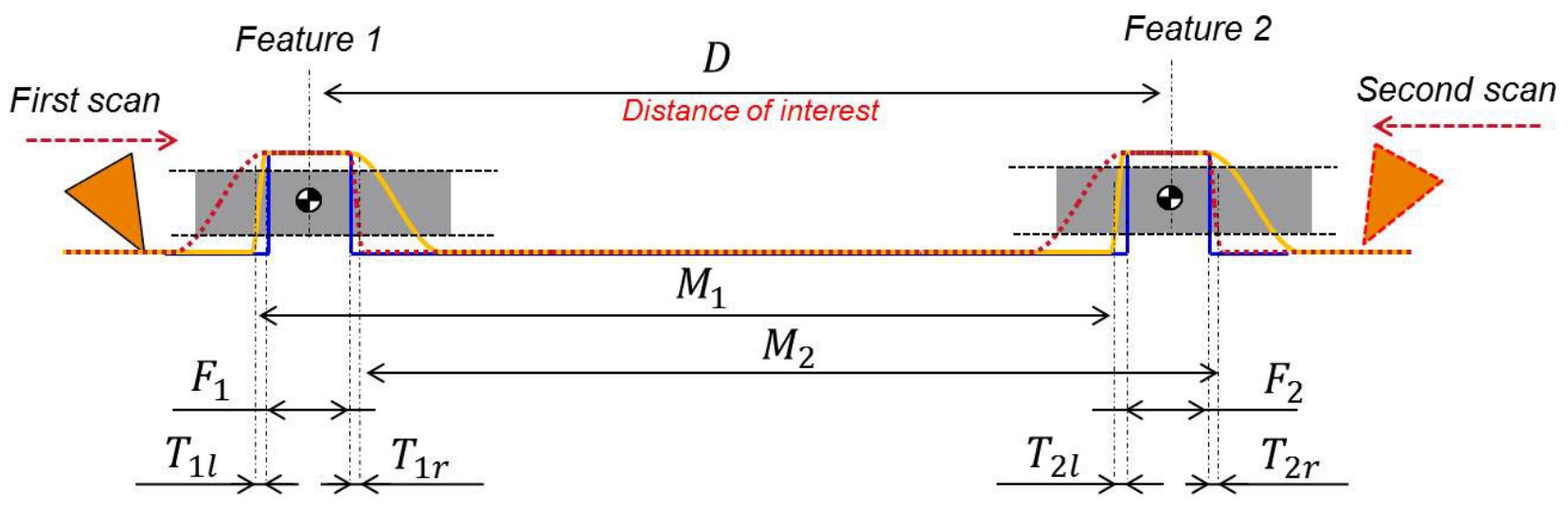

Figure 3: Sketch of the AFM based measurement procedure, The AFM probe is over tilted to one side, to allow contact with the steep sidewalls of the features. Two measurement is repeated twice while the probe is rotated such that both sidewalls of the features can be fully measured.

To overcome these problems, in this concept the AFM probe is over tilted in one direction, as depicted in Figure 3. Since the probe is tilted more than the half cone angle and the possible overhang of the structure, one sidewall of the feature can be fully probed with the sharp end of the probe. Subsequently, the measurement is repeated while the probe is rotated 180 degrees with the sample to measure the adjacent sidewall with the same sharp edge of the probe. As a result two feature to feature measurement are obtained: one measurement allowing to accurate determine the distance from the left flanges of both features, denoted $\mathrm{M}_{1}$ in figure 3 , and one measurement allowing to determine the distances between te right flanges, denoted $\mathrm{M}_{2}$ :

$$
\begin{aligned}
& M_{1}=D+1 / 2 \cdot F_{1}-1 / 2 \cdot F_{2}+T_{1 l}-T_{2 l} \\
& M_{2}=D-1 / 2 \cdot F_{1}+1 / 2 \cdot F_{2}-T_{1 r}+T_{2 r}
\end{aligned}
$$

with all parameters as defined in Figure 3. Since the features on semiconductor devices are largely symmetric and both sides of the features are measured with the same side of the probe, the broadening of the features due to the tipconvolution is about equal on both sides: $T_{1 l} \approx T_{1 r}$, and $T_{2 l} \approx T_{2 r}$. Hence, by taking the average of both measurements the actual distance of interest is obtained:

$$
D=\frac{M_{1}+M_{2}}{2}
$$

In practise the features in semiconductor devices may have a certain amount of asymmetry, typically up to about $5^{\circ}$ in sidewall angle. This causes the left and right part of the feature to contact a different part of the probe, and as a result the broadening of the features is not the same at both sides, causing a slight position measurement error. However, when using the ultra-sharp AFM probes with tip-radius down to about $2 \mathrm{~nm}$, the resulting position measurement error is in the order of only a few picometer. 
From a practical point of view, doing the measurement twice while rotating the probe requires breaking up the metrology loop. As a result the tip location with respect to the metrology coordinate system is not preserved in between both measurements. However, this is not a problem since both measurement involve a relative marker to feature measurement and the absolute probe position between both measurement is not of interest.

AFM imaging with over tilted AFM probes has been successfully demonstrated by several groups [2,4]. An additional advantages of this technique is that is allows to fully characterize the profile of the marker features. The information on the marker profile could be used to predict the position interpretation of optical diffraction based alignment sensors used in the lithography machine. These alignment sensors are sensitive for the asymmetry of the marker features, which can cause an offset in the marker position interpretation of these sensors. These position offsets could be predicted and compensated for based on the measured marker asymmetry with AFM.

\section{LARGE DYNAMIC RANGE AFM DEVELOPMENT}

Given the above mentioned application, an AFM system is needed that is capable of measuring the marker to feature distances over several millimeters with sub-nanometer total measurement uncertainty. Hence, the AFM system should be able to position the probe relative to the wafer over several millimeters distance, and keep track of the of this motion with sub-nanometer accuracy. In other words, an dynamic measurement range is needed in the order of $10^{7}$, which is the major challenge of this project. Most current available AFM systems have positioning ranges in the order of 10-100 $\mu \mathrm{m}$ with accuracy levels of around $1 \%$ over that range, and thus are by far not capable of meeting these requirements. Several studies can be found in literature on the development of highly accurate AFM systems with long positioning ranges [1,5], but this technology is not very mature and widely available. Hence, to fulfill these requirements a whole new AFM system has to be developed, which is the main focus of this research program.

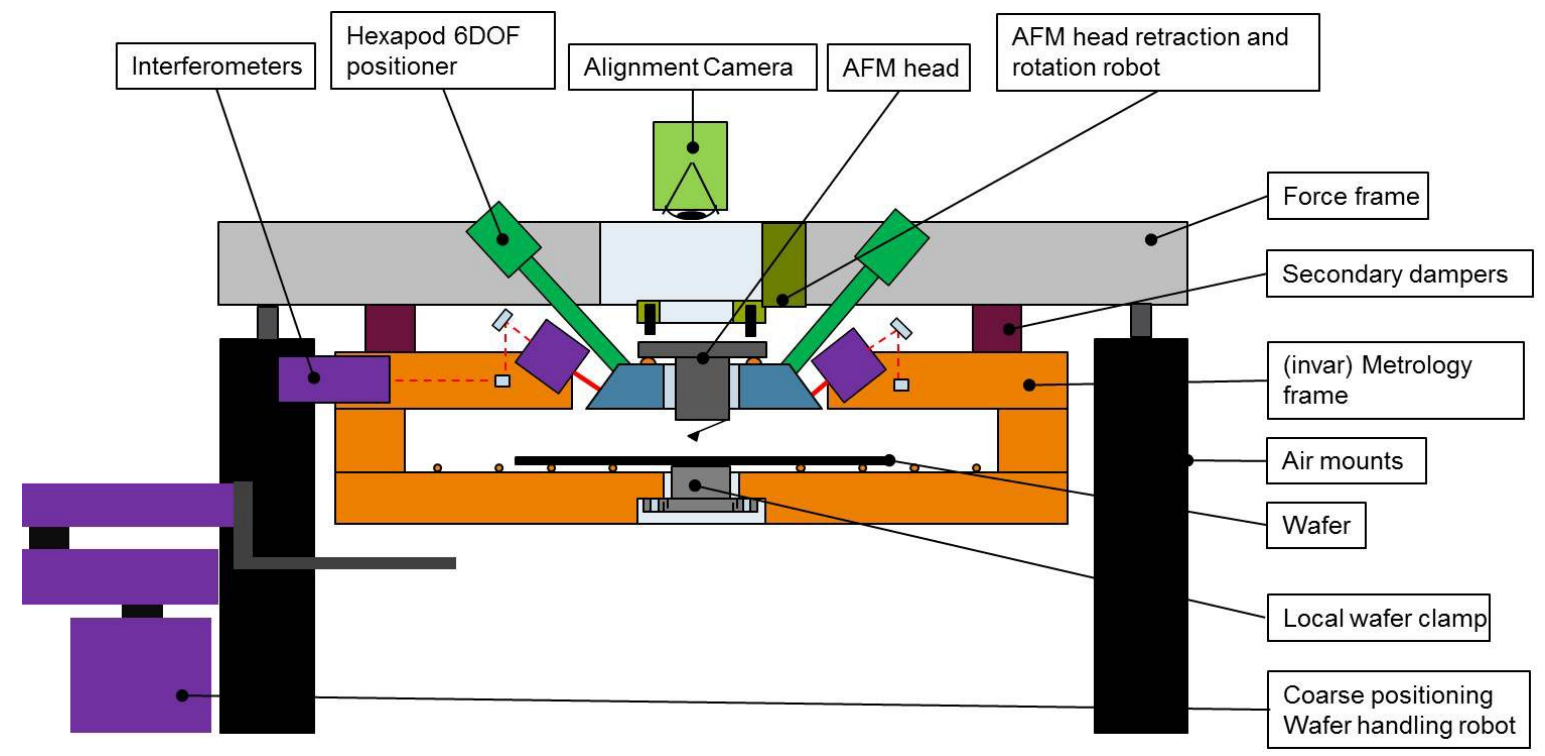

Figure 4: Conceptual drawing of the large dynamic range AFM under development at TNO.

\subsection{System architecture}

The AFM system under development at TNO is depicted schematically in Figure 4. The core of the system is the AFM head, containing the AFM cantilever, and an Optical Beam Deflection sensor to measure the deflection of the cantilever. The AFM head is position with respect to the wafer via an hexapod positioning stage. The stage displacement is measured with interferometers with respect to a metrology frame. The metrology frame is connected to the wafer clamp which rigidly holds the wafer with the field of interest underneath the AFM probe. An wafer handling robot is used to 
position the wafer from the cassette into the machine, and to position the wafer with the right field of interest underneath the AFM probe. An additional robot is used to extract the AFM head out to the positing stage to allow exchange of the AFM probe, or to rotate the AFM head with respect to the sample as to part of the measurement procedure described in Section 2.3. Air mounts are used to isolate the entire system from ground vibrations.

In order to achieve the required measurement accuracy two aspects needed special attention: minimizing the Abbe-error, and minimizing the drift over the metrology loop. In the subsequent sections these aspects are further explained.

\subsection{Minimization of Abbe errors}

In principle the AFM probe is positioned in the three translational directions with respect to the sample, while the orientation of the probe is intended to remain constant. However, slight rotations of the positioning stage can occur during the AFM scanning which can result in Abbe-errors when the measurement axes are not directly in line with the probe. To minimize the Abbe error, this system is designed with the aim of minimizing both the measurement offset and the stage rotations during scanning.

The position and orientation of the stage is measured with respect to a metrology frame using $32 \mathrm{DOF}$ heterodyne interferometers, which are customized versions of the Keysight E1827A 2-Axis Interferometer. The measurement lines of the interferometers are all aligned towards the probe in an orthogonal configuration, radiating $120^{\circ}$ about the vertical axis and $54.74^{\circ}$ about the normal. The target mirror is shown in Figure 5. Similar configurations of the interferometers can be found in $[6,7]$. The result of this configuration is minimal offset between the measurement axes and the probe.

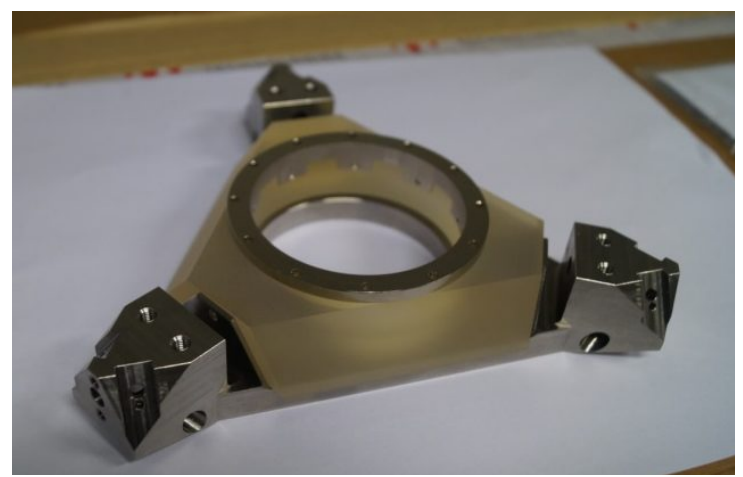

Figure 5: Picture of the (yet unpolished) target mirror. The titanium frame connects this mirror to the legs of the hexapod stage. The central invar ring is the interface with the AFM-head, which is clamped via magnets.

While the stage metrology is designed to minimize the offsets between the measurement axes and the AFM probe, this offset can still reach several millimeters due to the translation of the probe with respect to the metrology system. An Abbe-offset of a few millimeters requires the orientation of the probe to be stable in the order of $0.1 \mu$ rads for the Abbe error to be below $1 \mathrm{~nm}$. This kind of stability is deemed not feasible with an mechanical linear guidance over the required positioning range, and therefore its decided to control the probe in 6 degrees of freedom, where the orientations are controlled to be constant during the AFM measurements. A similar 6DOF control approach in a metrological AFM systems can be found in [8].

To enable 6 DOF control of the AFM head in combination with the long traveling ranges, a hexapod positioning stage is developed. The hexapod is driven by 6 voice-coil actuators which are guided by air-bearings, as depicted in Figure 6 . On one side the voice-coil actuators connect to the moving platform via flexural hinges, while on the other side they connect to a force-frame consisting of a large aluminum block. Due to the low stiffness of the stage suspension and the airbearing guidance, the first mechanical resonance frequencies of the stage are low which results in effective filtering of vibrations from the force frame and electrical noise in the voice-coil actuators. To minimize the heat generation of the voice-coils, the gravity forces acting on the stage are compensated via a magnetic gravity compensator. The lateral positioning range of the stage is a hexagonal area with minimal width of $8 \mathrm{~mm}$, and the vertical positioning range is $4 \mathrm{~mm}$. 


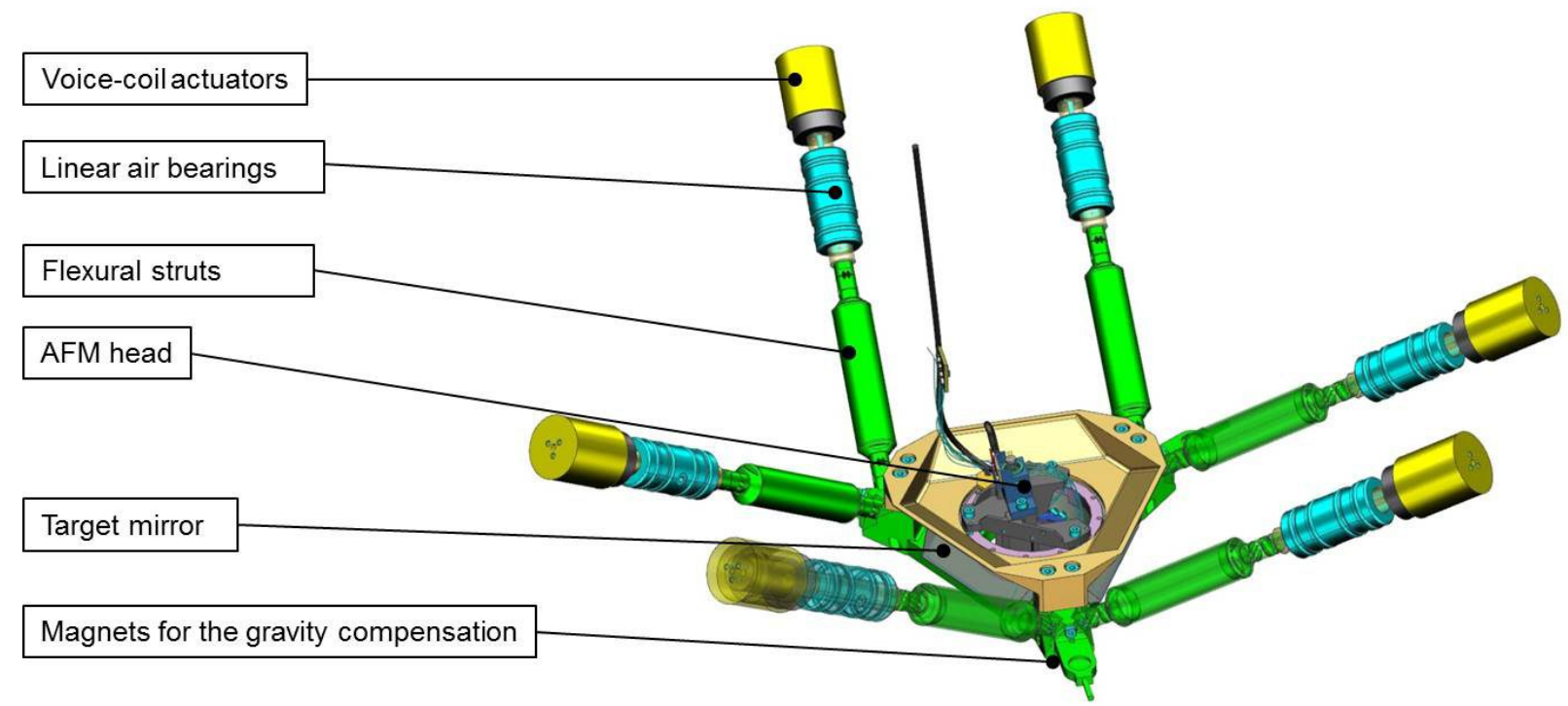

Figure 6: CAD drawing of the hexapod positioning stage.

\subsection{High stability metrology concept.}

Since in AFM the features are probed point by point, AFM imaging is a relatively slow process. It is estimated that the marker to device feature measurement can take up to about 2 minutes if several device features are measured after each other. To ensure the sub-nanometer total measurement uncertainty the metrology loop is required to be highly stable over the course of this measurement time. What makes this extra challenging is that the size of the $300 \mathrm{~mm}$ wafer results in a relatively large metrology loop, from the area of interest on the wafer all the wat towards the measurement probe. Most AFM systems are built as compact as possible to minimize the susceptibility to drifts, but for this instrument this is not possible.

To meet such high stability requirements a novel metrology concept is developed. The metrology frame is made of invar, and rigidly connects the interferometers with the vacuum wafer clamp, as depicted in Figure 7. The wafer clamp is significantly smaller in diameter than the full wafer, and only clamps the wafer locally with the field of interest underneath the AFM probe, as depicted in Figure 4. The outer ends of the wafer are supported in the vertical direction, but are able the expand in the lateral direction. As a result the wafer is always thermally centered with the field of interest underneath the measurement probe, which significantly contributes to the thermal stability of the system. Positioning of the wafer with the right field of interest is done with the wafer-handling robot. After positioning the wafer the wafer handling robot is retracted, and the wafer is clamped down with the vacuum clamp. Hence, the coarse wafer positioner is outside the metrology loop during the AFM measurements, which significantly minimized the drift sources in the metrology loop.

The entire system will be housed in a enclosure with a separate air-conditioning unit to keep its temperature as constant as possible. 


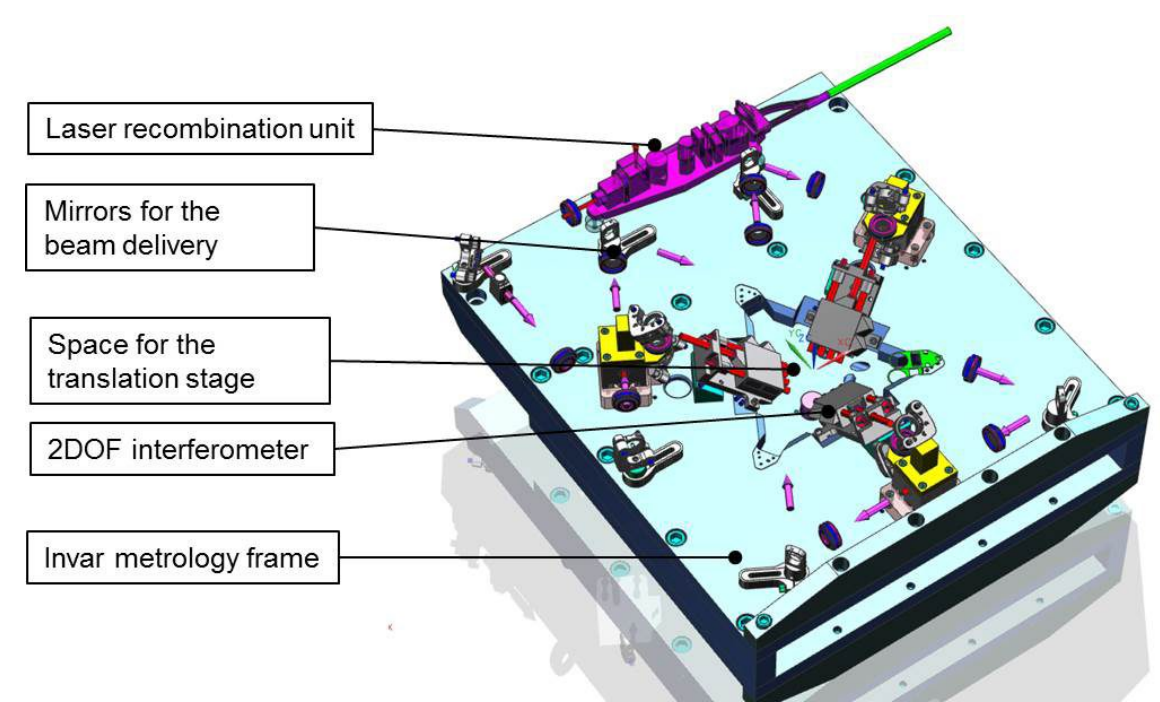

Figure 7: CAD drawing of the metrology frame with the laser beam delivery, and three 2DOF interferometers.

\section{DEVELOPMENT PLAN \& INITIAL TEST RESULTS}

In measuring the marker to device feature distance with the developed AFM system, the error contributions can be separated in different factors:

a. Errors due to drifts: thermal drift in the mechanical structure, and in the interferometric measurement due to changes in the refractive index of air, and potential wear of the AFM probe.

b. Random error contributions due to environmental noise, electrical noise, and the line-edge roughness of the sample

c. Non-linearity errors: due to unflattness of the target mirror, clamp distortion of the wafer grid, non-linearity of the interferometry measurement, and errors due to assymetric probe convolution.

Notice that systematic calibration errors and errors due to temperature variations are compensated for by scalling the measured feature to marker distance relative to the marker grid, as discussed in Section 2.

To verify the feasibility of this measurement concept a demonstrator system is currently being build. The first goal of this demonstrator is to show the capability of measuring a marker to feature distance of several millimeters with subnanometer measurement repeatibility. If this measurement repeatibility is met this means that the abovementioned drift and random error contributions are sufficiently under control. Once the measurement repeatibility is demonstrated the non-linearity error contributions will be addressed and verified.

As a first step in realizing this demonstrator system, the AFM-head with the cantilever holder and optical beam deflection (OBD) sensor are build and tested. Since this component is the core of the entire system, it is build and tested first before the rest of the system is designed around it. Figure 8 shows the results of the cantilever deflection test, clearly revealing the cantilever oscillations at $250 \mathrm{kHz}$. This means that the noise of the cantilever deflection measurement is dominated by the inevitible thermal noise of the cantilever, and that the (electrical/optical) background noise is well below this level. Hence, based on these test the performance of the OBD is verified.

Another design driving element is the alignment camera and its illumination. This camera is used to initially find the location of the probe with respect to the aligment features to allow bringing the probe close to the marker feature before starting the actual AFM measurement. The main aspect to be verified with these test is whether the illumination from the top side results in sufficient contrast between the probe and the marker features. The illumination is brought in via a beamsplitter below the camera unit. Figure 9 shows the result of these camera tests, clearly revealing the AFM probe and the marker features of the semiconductor device.

The next steps are the integration of the full AFM system demonstrator to start the rest of the abovementioned test campaign. 


\section{CONCLUSIONS}

In this paper we have presented the development of a large dynamic range AFM system to enable overlay improvements. By measuring the exact location of the functional device features with respect to the alignment marker grid within each layer, overlay errors stemming from position errors between the coarse alignment marker and the much finer device features can be compensated for.

The AFM system under development has several unique characteristics:

- a very large measurement range of $8 \times 8 \mathrm{~mm}$ (hexagonal)

- sub-nanometer measurement uncertainty over this large measurement range,

- $\quad$ the capability of measuring large substrates with high thermal stability (e.g. wafers/reticle masks),

- the capability to fully profile high aspect ratio features from multiple sides

Besides the application of overlay improvements, these unique characteristics may address several applications in the fields of semiconductor manufacturing and nano-metrology. For instance, the instrument may be used for the calibration and characterization of grid patterns on calibration wafers, or the calibration of grid plates used for optical encoder based metrology. In fact, the need for such large dynamic range AFM systems is highlighted in several nanometrology roadmaps [9].

\section{REFERENCES}

[1] Kato, T., et al. "Fundamentals of overlay measurement and inspection using scanning electron-microscope." SPIE Advanced Lithography. International Society for Optics and Photonics, 8681 86812Q-1, (2013)

[2] Danzebrink, H-U., et al. "Advances in scanning force microscopy for dimensional metrology." CIRP AnnalsManufacturing Technology 55(2), 841-878 (2006):

[3] Foucher, J., et al. "Introduction of next-generation 3D AFM for advanced process control." SPIE Advanced Lithography. International Society for Optics and Photonics, 868106-868106-8 (2013)

[4] Sadeghian, H., et al. "Parallel, miniaturized scanning probe microscope for defect inspection and review." SPIE Advanced Lithography. International Society for Optics and Photonics, 90501B-90501B (2014).

[5] Murayama, Ken, et al. "Side-wall measurement using tilt-scanning method in atomic force microscope." Japanese journal of applied physics 45(6S), 5423 (2006)

[6] Jäger, G., et al. "Nanomeasuring and nanopositioning engineering." Measurement 43.9 (2010): 1099-1105.

[7] Werner, Chris, P. C. J. N. Rosielle, and Maarten Steinbuch. "Design of a long stroke translation stage for AFM." International Journal of Machine Tools and Manufacture 50(2), 183-190 (2010)

[8] Ducourtieux, Sebastien, and Benoit Poyet. "Development of a metrological atomic force microscope with minimized Abbe error and differential interferometer-based real-time position control." Measurement Science and Technology 22(9), 094010 (2011)

[9] Lazar, Josef, et al. "Short-range six-axis interferometer controlled positioning for scanning probe microscopy." Sensors 14(1), 877-86 (2014)

[10] Euramet, Science and technology roadmap for metrology (2012) 


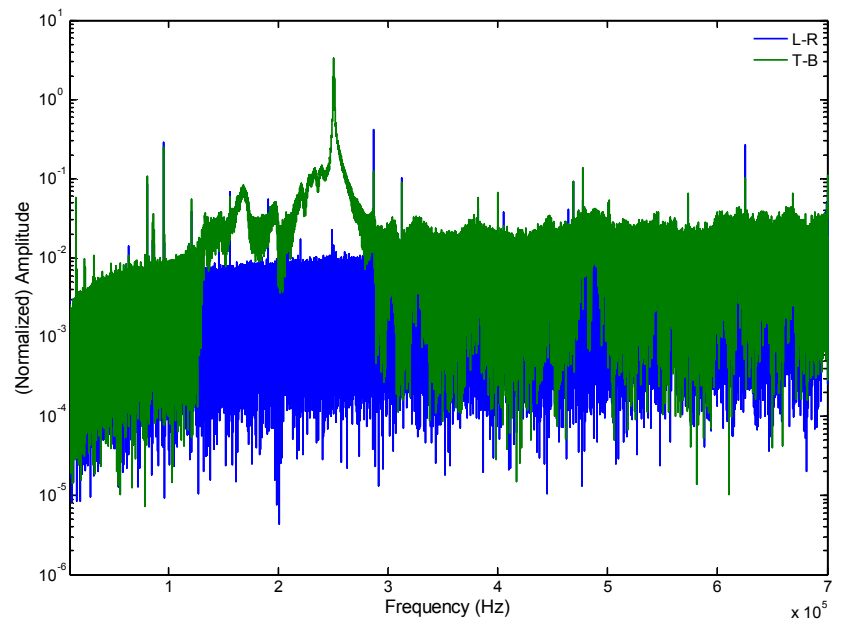

Figure 8: Test results of the optical beam deflection measurement, clearly revealing the thermal noise of the cantilever at its resonance frequency of $250 \mathrm{kHz}$ standing out from background noise.

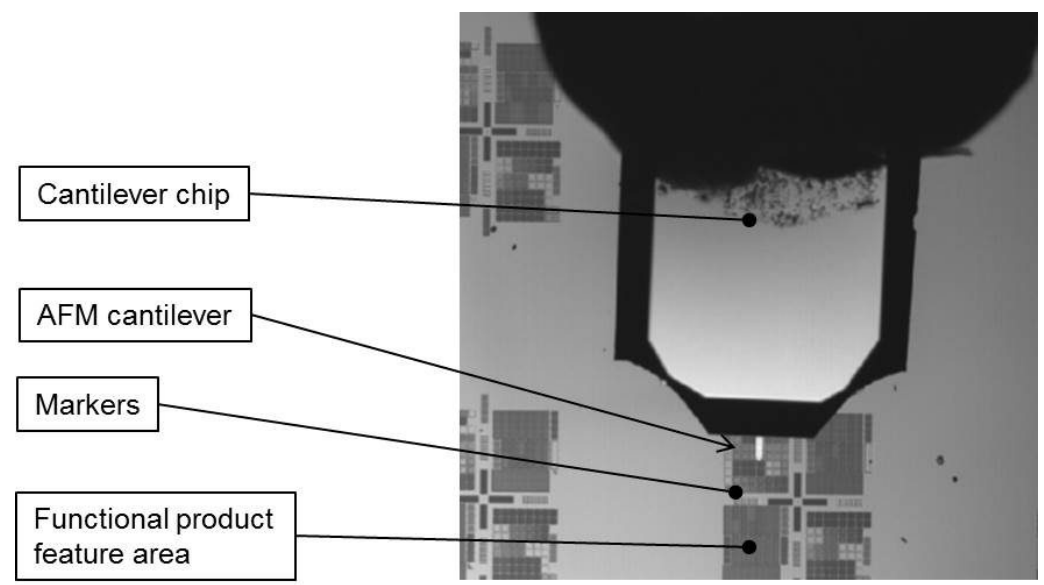

Figure 9: View of the alignment camera, revealing both the AFM cantilever and the marker features. 